\title{
Effects of delayed presentation of intussusception in infants and children
}

\author{
Mohamed Fathy Metwally, MD
}

Department of Surgery, Tanta University, Egypt.

\begin{abstract}
Background/purpose: Many children with intussusception are reported to present late for definitive therapy. Attempted nonsurgical reduction of intussusceptions after 48 hours is controversial because of the low probability of reduction and an increased risk of perforation. This study was conducted to determine the effect of delayed presentation on clinical parameters, management, and the outcome of childhood intussusceptions. The author also studied the diagnostic investigations that can give criteria that may help to predict bowel viability and to choose the most suitable management.

Methods: Comparative analysis of 44 children with intussusception managed from 2007 to 2011 at Tanta university and Saudi Arabia was done.

Results: The mean time from onset of presentation was 3 days (range from 4 hours to 7 days). Thirty seven patients presented within 48 hours of symptoms (group 1) and 7patients presented after 48 hours (group 2). Clinical presentations were similar in the children with the exception of bilious vomiting, rectal bleeding, and abdominal distension that were significantly commoner in group 2 children $(p<0.05)$. The type of intussusceptions found at operation did not differ in the groups, but bowel complications and the incidence of failed reduction and bowel resection were higher in group 2 patients $(p<0.05)$. Though the postoperative complications did not differ significantly between the two groups, mortality directly related to intussusceptions occurred only in patients who presented after 48 hours.

Conclusion: Significant number of children with intussusceptions present late for definitive treatment. These cases have a high risk of bowel complications and intestinal resection. The outcome of these patients can be improved through good perioperative care and reducing delays in seeking health care. Also, cases of delayed presentation can safely undergo contrast enema reduction if no bowel-wall edema of the intussuscipiens or obstruction or ischemia is demonstrated.
\end{abstract}

Key words: Childhood, intussusceptions, delayed presentation, outcome.

\section{Introduction:}

Intussusception is a common cause of gastrointestinal obstruction in infants and young children $(15 \%) .{ }^{1}$ The peak incidence is between 4 and 6 months with a male to female ratio of $1.3: 1 .^{2-4}$ Clinical presentations are well known that definitive diagnosis and management in most cases of intussusception is expected to be done within 48 hours of onset of symptoms. The presenting features of intussusception are sudden onset of intermittent colicky abdominal pain in a previously well child. Infants and children may strain, draw their knees up, act very irritable, and cry loudly. The child may recover and become playful inbetween bouts of pain, or become tired and weak from crying. Vomiting usually starts soon after the pain begins. Abdominal distention and constipation are common, although the child may pass normal stools initially, the following stools may look bloody with mucus giving 
jell-like stools. Palpable abdominal mass and sometimes, palpable mass on rectal examination may be felt. ${ }^{2-7}$ In spite of this, a considerable number of children with intussusception, for ill-defined reasons, present late for treatment. This delayed presentation might be a significant cause for the poorer outcome of treatment in these cases. ${ }^{8}$

Intussusception is diagnosed by radiology, ultrasonography, Doppler, and surgery. Abdominal radiography may show dilated small bowel and absence of gas in the region of the cecum. In some cases, a mass impression within the colonic gas with an "air crescent" sign from air trapped between the bowel walls, indicates an intraluminal mass created by the intussuscepting loop. Sometimes, radiographs may appear indeterminate or normal, therefore, the presence of an unremarkable abdominal radiograph should not be the base for excluding a diagnosis of intussusception. ${ }^{9}$ Contrast and pneumatic enemas have dual role in diagnosis and treatment of intussusception. Water - soluble radiopaque agents as well as air are used for enemas as they show coiled spring appearance with arrest of barium at the end of intussusception. ${ }^{1}$

Ultrasonography has now proved its accuracy for diagnosis of intussusception. Transverse ultrasonogram shows a mass with a swirled appearance of alternating sonolucent and hyperechoic bowel wall of the loop-within-a-loop. On longitudinal ultra sonograms, the intussuscipiens and the intussusceptum have the appearance of a submarine sandwich or pseudo kidney sign.

There appear to be multiple layers, which represent the walls of the intussuscepted bowel loops. Although ultrasonographic examination is almost always positive, overlying loops of air-containing bowel may obscure intussusception. ${ }^{9}$ Some authors found that hydrostatic reduction was unlikely or impossible when the outer rim of the intussusception target sign on ultrasonic was more than $10 \mathrm{~mm}$ thick or when a large amount of free peritoneal fluid was found. ${ }^{10}$ Other authors showed no correlation between the edema, peritoneal fluid or of the occlusive syndrome and the severity of ischemia. ${ }^{10}$
In spite of this, clinical and radiological findings are unable to predict the intensity of the vascular injury.

The invagination of a segment of bowel induces a mechanism of strangulation where as the intussusceptum proceeds distally, its mesentery becomes stretched, angulated and compressed between the layers of the bowel wall, first causing partial inhibition of venous drainage, venous congestion and edema that contribute to tighten the intussusceptum and obstruct the small bowel. A second phase of arterial occlusion will occur with hemorrhage, gangrene and risk of perforation. Severe ischemia is often encountered in ileoileal and trans-valvular ileocolic types, where the mesentery is short and the intussusceptum has a narrow neck. ${ }^{11}$

In intussusceptions, color Doppler allows an interesting evaluation of the degree of bowel ischemia and necrosis, can change the therapeutic management and appears promising for appreciating the viability of the occluded bowel loops. ${ }^{11}$ $\mathrm{CT}$ is generally the imaging modality of choice as the images on CT are pathognomonic for intussusception. A CT scan may have a further advantage by providing clues to the etiology of the intussusception as lymphadenopathy or malignant lesions. ${ }^{1}$ Many recent studies on intussusception have paid great attention to children who present after 48 hours of symptoms, in a trial to develop feasible initiatives for better early diagnosis and better results of treatment for the patients. ${ }^{5-7,12,13}$ Treatment includes air or hydrostatic reduction enema under radiologic or ultrasound guidance, surgery reduction (traditional and laparoscopic) and in approximately $10 \%$ of cases intestinal resection due to intestinal ischemia. ${ }^{14}$

\section{Patients and methods:}

This study was conducted at Tanta University Hospital and Saudi Arabia, over a period of 4 years from 2007 to 2011. Forty four patients were managed for intussusception. Diagnosis was based mainly on clinical, radiological, ultrasound and Doppler evaluation. All cases received 
fluid and electrolyte resuscitation. Definitive treatment including operative intervention was done in all cases.

For the purpose of this study, delayed presentation was defined as presentation after 48 hours of onset of symptoms. The clinical features, operative treatment, complications and outcome were compared between the cases presented after 48 hours and those presenting earlier, in order to determine the effect of delayed presentation on these variables.

At the start of the study the author selected operative intervention for all cases (15 cases) to correlate between the clinical features, radiological investigations and operative findings. Later, the author started to use other methods of management as hydrostatic reduction, in addition to the operative intervention.

Color Doppler has been attempted to determine the intensity of ischemia and the possibility of reducing intussusception in all cases. In group 1, there were evidence of venous \& arterial hyperemia within the intussusceptum in 37 cases. Complete reduction by hydrostatic reduction was achieved in 7 cases (19\%), simple reduction by milking after surgical exploration in 22 cases (59\%), simple laparoscopic reduction in 5 cases $(14 \%)$ and resection anastomosis was required in the other 3 cases $(8 \%)$. The indication for resection in these 3 cases was failure of simple reduction due to severe edema.
Considering the 7 cases in group 2, there was hyperemia in 2 cases $(29 \%)$ and 5 cases $(71 \%)$ without hyperemia. No attempt for hydrostatic or pneumatic reduction was done for all cases and surgical intervention was done immediately. In the 2 cases with hyperemia, surgical reduction was done with some difficulty with the aid of hot fomentations. The other 5 cases $(100 \%)$ required resection anastomosis due to the marked ischemia and necrosis of the intestine.

\section{Results:}

The 44 children included 37 patients $(84 \%)$ who presented within 48 hours of symptoms (group 1) and 7 patients (16\%) who presented after 48 hours of symptoms (group 2). Thirty patients (68\%) were males and $14(32 \%)$ were females. The median age at presentation was 7 months (range 3 months -3 years).

All cases presented with abdominal pain. The other features varied in the two groups. In group 1, the presenting features included bilious vomiting 14 cases (38\%), rectal bleeding 11 cases $(30 \%)$, abdominal distension 14 cases (38\%), palpable abdominal mass 17 cases (46\%), and silent abdomen 3 cases (8\%). The clinical features in group 2 patients included bilious vomiting 6 cases $(86 \%)$, rectal bleeding 5 cases (71\%), abdominal distension 5 cases $(71 \%)$, palpable abdominal mass 4 cases $(57 \%)$, and silent abdomen 2 cases $(29 \%)$.

\section{Table(1): Shows the comparison between the clinical presentation of intussusception in children presenting early and those presenting after 48 hours.}

\begin{tabular}{|l|c|c|c|}
\hline Clinical features & \multicolumn{2}{|c|}{ Duration of symptoms } & \\
\hline & $\begin{array}{c}\text { Less than 48 hours } \\
(\mathrm{n}=37)\end{array}$ & $\begin{array}{c}\text { More than 48 hours } \\
(\mathrm{n}=7)\end{array}$ & \\
\hline Abdominal pain & $37(100 \%)$ & $7(100 \%)$ & - \\
\hline Bilious vomiting & $14(38 \%)$ & $6(86 \%)$ & $0.001^{*}$ \\
\hline Rectal bleeding & $11(30 \%)$ & $5(71 \%)$ & $0.001^{*}$ \\
\hline Abdominal distension & $14(38 \%)$ & $5(71 \%)$ & $0.024^{*}$ \\
\hline Palpable abdominal mass & $17(46 \%)$ & $4(57 \%)$ & 0.729 \\
\hline Silent abdomen & $3(8 \%)$ & $2(29 \%)$ & 0.294 \\
\hline
\end{tabular}

* Statistically significant $(p<0.05)$ 
The average duration of symptoms before presentation to the hospital was 3 days (range 4 hours - 7 days).

At operation, cases in group 1 had ileocolic intussusception in $28(76 \%)$, ileoileal in $6(16 \%)$, and colocolic in $3(8$ $\%)$. Children in group 2, had ileocolic type in6 (86\%) and colocolic one patient (14\%). Successful reduction of intussusception was done in 34 cases (92\%) in group 1 (hydrostatic reduction in 7 cases, traditional surgical reduction in 22 cases and laparoscopic reduction in 5 cases), compared to 2 cases $(29 \%)$ in group $2(\mathrm{p}=0.03)$ by traditional surgical reduction. In the other 8 patients, the intussusception was either irreducible in 3 patients in group 1, or was with necrotic bowel in 5 patients in group 2 . The eight cases had right hemicolectomy ( 3 cases in group 1 and 5 cases in group 2).
All these cases were of the ileocolic type. All surgical wounds were closed primarily. None of the patients was managed in an intensive care unit. Postoperatively, the complications were superficial wound infection in 3 cases, adhesive small bowel obstruction in one case and chest infection in 3 cases.

Superficial wound infection was managed by local wound care. The case of adhesive bowel obstruction responded to conservative management.

Follow up was done for an average of 10 months (range 3-29 months). There was no recurrence of intussusception and the postoperative complications did not differ significantly between the two groups. One patient $(2 \%)$ died at the tenth day after the operation due to septicemia in group 2.

\section{Table (2): Shows comparison between the postoperative complications in the 2 groups.}

\begin{tabular}{|l|c|c|}
\hline Clinical features & \multicolumn{2}{|c|}{ Duration of symptoms } \\
\hline & $\begin{array}{c}\text { Less than 48 hours } \\
(\mathrm{n}=37)\end{array}$ & $\begin{array}{c}\text { More than } 48 \text { hours } \\
(\mathrm{n}=7)\end{array}$ \\
\hline Surgical wound infection & - & 3 \\
\hline Adhesive bowel obstruction & - & 1 \\
\hline Chest infection & 1 & 2 \\
\hline Death & - & 1 \\
\hline
\end{tabular}

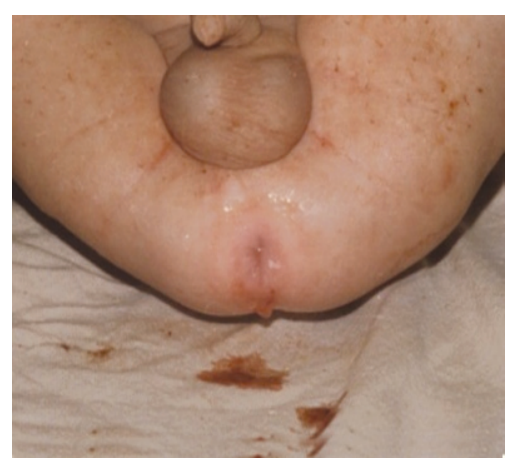

Figure (1a): A case of Intussusception showing the passage of red currant jelly.

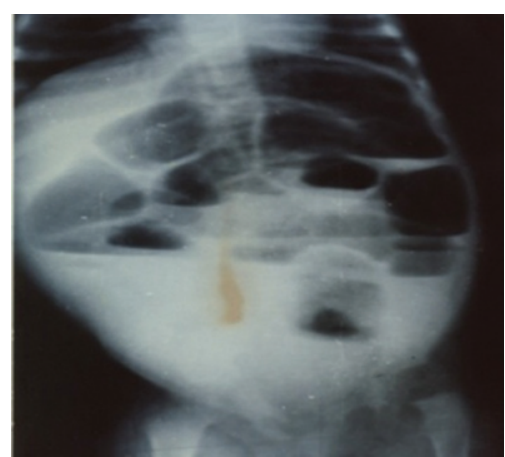

Figure (1b): Plain $x$-ray abdomen (erect) showing multiple air and fluid levels in the same case of intussusception.

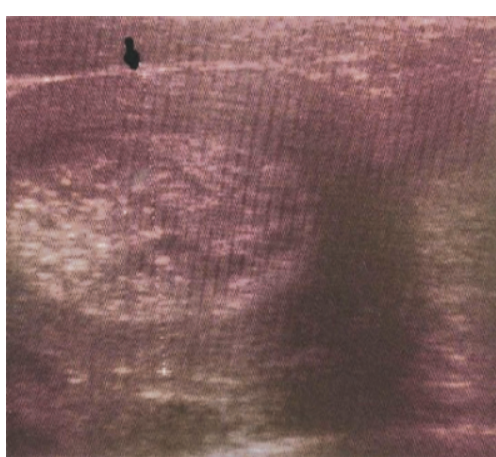

Figure (1c): US of the same case showing intussusception with enlarged mesenteric lymph nodes. 


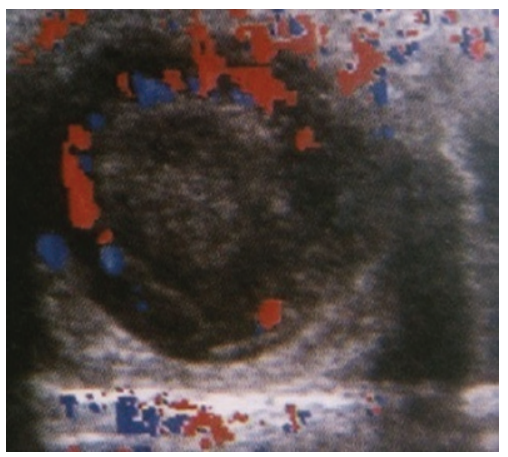

Figure (1d): Color Doppler of the same case showing marked hyperemia, indicating viable intestine.

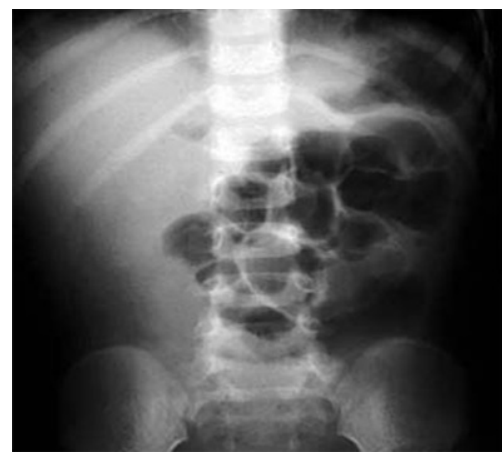

Figure (2a): Plain x-ray of a case of intussusception showing absence of cecal air and an obstruction pattern are seen.

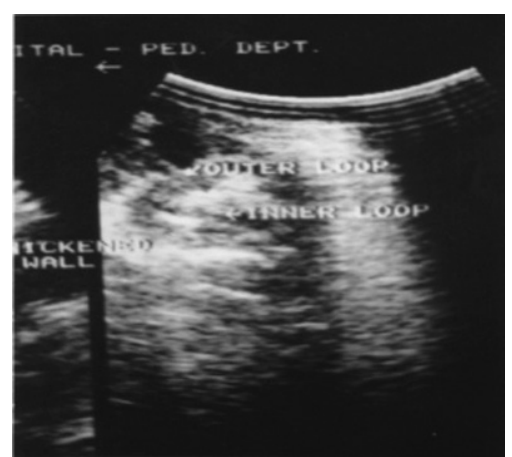

Figure (3a): US of another case showing target like lesion in a case of ileocolic intussusception .

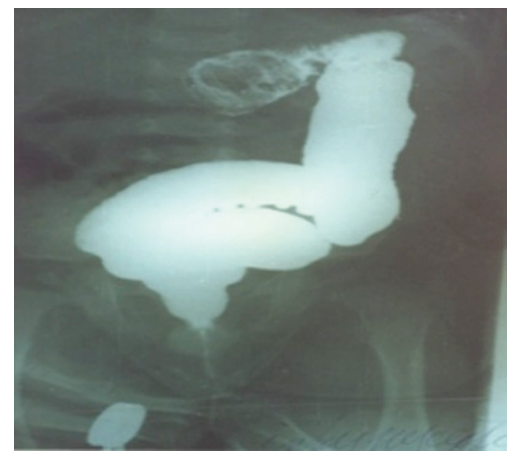

Figure (1e): Barium enema showing coiled spring appearance with arrest of barium at the end of intussusception.

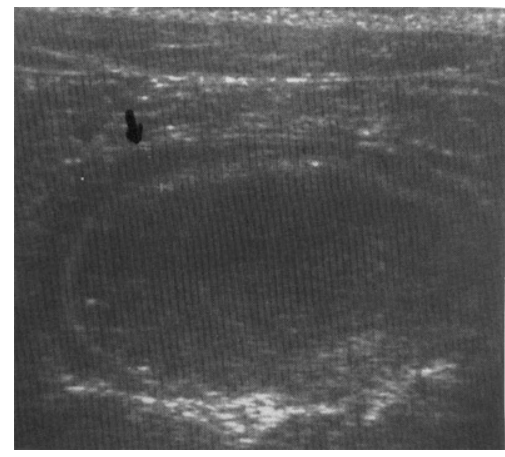

Figure (2b): US of the same case showing intussusception.

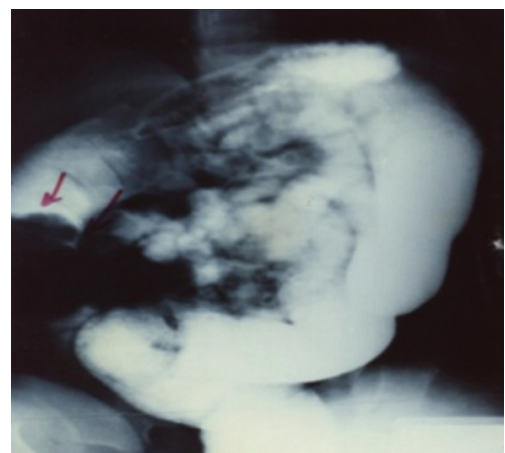

Figure (3b): Barium enema of the same case showing arrest of the barium at the hepatic flexure.

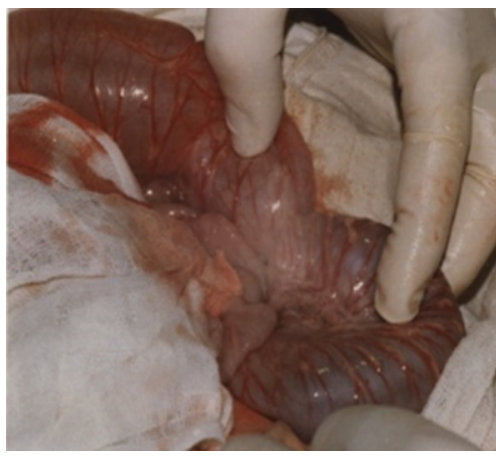

Figure (1f): Intraoperative picture showing colocolic intussusception with viable intestine, treated by simple reduction.

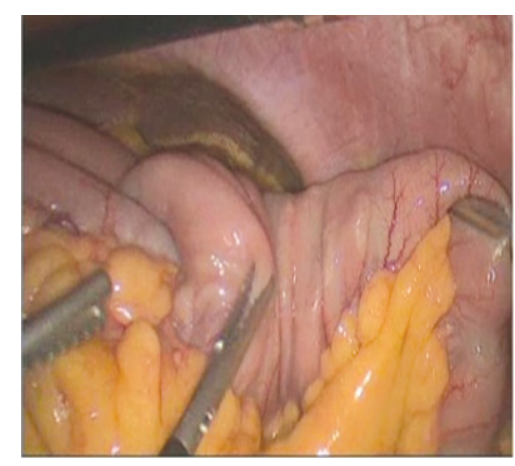

Figure (2c): Laparoscopic picture of the patient with no edema or ischemia showing simple reduction of the intussusception.

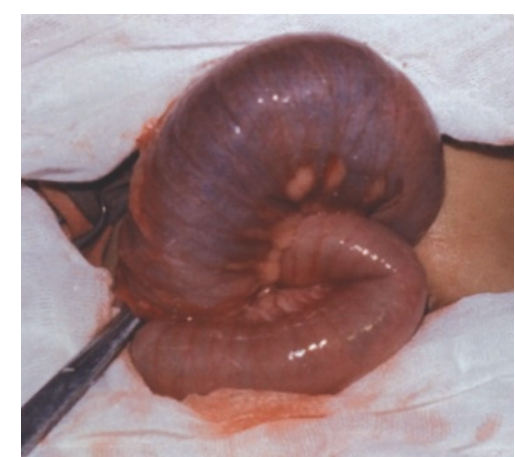

Figure (3c): Intraoperative picture of the same case showing ileocolic intussusception with viable intestine treated by simple reduction. 


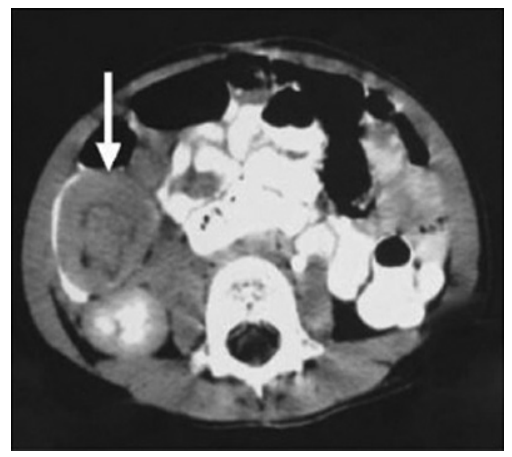

Figure (4a): CT scan of the patient showing the classic target sign in the sigmoid colon (arrow). There is no evidence of bowel wall edema of the intussuscipiens or of small bowel obstruction.

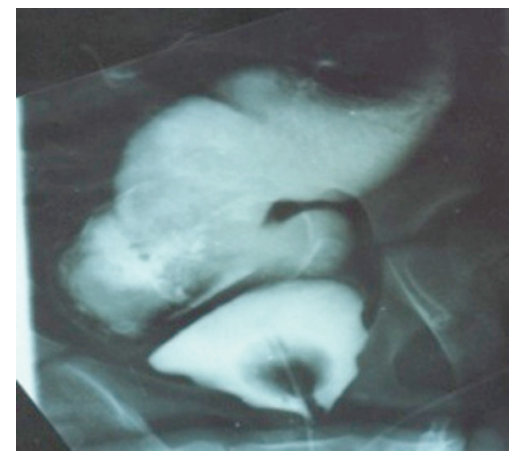

Figure (4b): $x$-ray showing hydrostatic reduction of the same case of colocolic intuss. with the mass in the sigmoid colon.

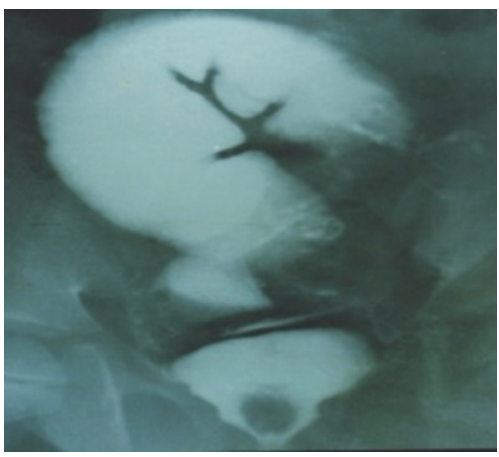

Figure (4c): Reduction of the mass into the descending colon.

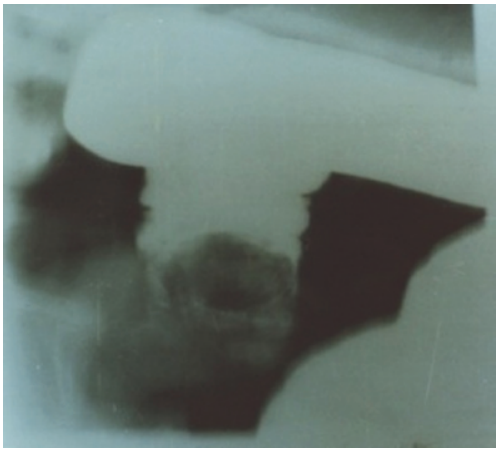

Figure (4d): Reduction of the mass into the ascending colon.

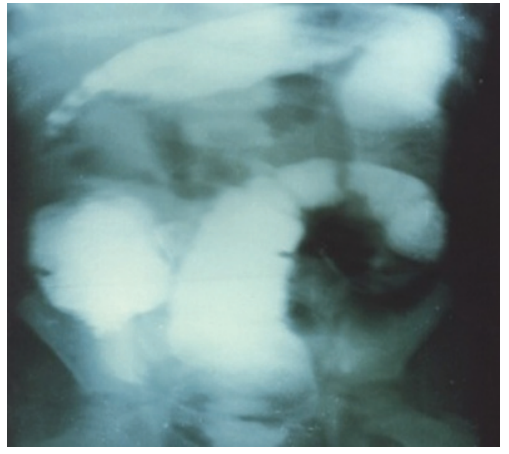

Figure (4e): Complete reduction of the intussusception.

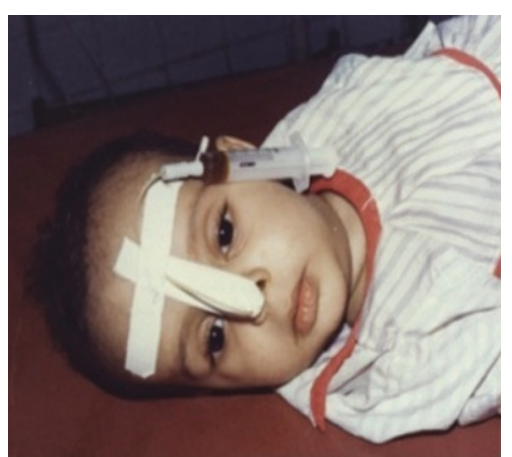

Figure (5a): A picture showing sunken eyes with feculent aspiration in a patient with delayed presentation of intussusception.

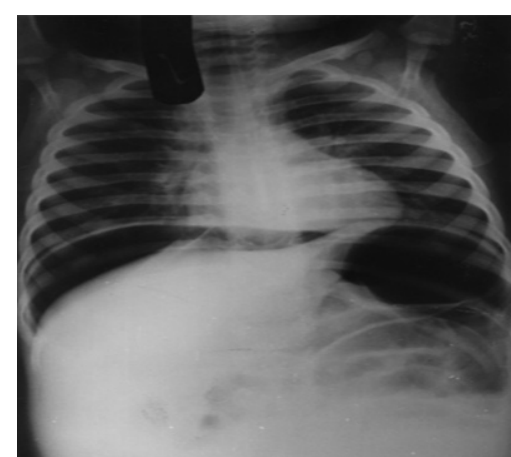

Figure (5b): Plain x ray abdomen (erect) of the same patient showing air under the Rt copula of the diaphragm due to perforated intestine.

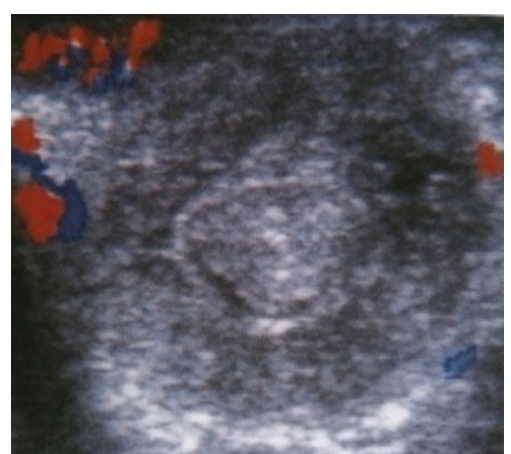

Figure (5c): Color Doppler of the same case showing sluggish arterial flow within the thickened wall of bowel. So, diagnosis of ischemic intestinal damage is highly suggested 


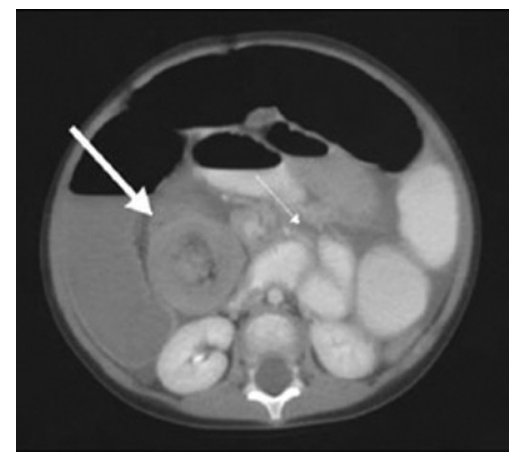

Figure (5d): CT scan of the patient showing the classic target sign (large arrow), bowel wall edema of the intussuscipiens ( small arrow) and dilated small bowel consistent with obstruction.

\section{Discussion:}

This study shows that some children with intussusception present for definitive treatment after 48 hours of symptoms. ${ }^{8,15-17}$ Many reports from developed countries indicate that the time of diagnosis and intervention in a majority of cases is less than 24 hours. $^{2}$ This may be due to ignorance, poverty and inadequate access to referral hospitals.

Previous reports have indicated that delayed presentation predisposes to bowel complications. ${ }^{6,13}$ This was reflected in the clinical presentation and the operative findings of the cases that presented after 48 hours of symptoms in this study.

Clinical features such as rectal bleeding, abdominal distension and absent bowel sounds which may be indicative of devitalized bowel, were commoner in children who presented after 48 hours. In the same time, a considerable number of irreducible and devitalized bowel found at operation in these cases, may confirm increased bowel complications with prolonged interval, from symptom onset to definitive treatment. This implies that one should have a high index of suspicion for the possible presence of devitalized bowel and adequately prepare such patients for bowel resection in case there is need for it. Operative findings indicate that the type of intussusception does not contribute to the delay in diagnosis.

Intussusception is currently treated by

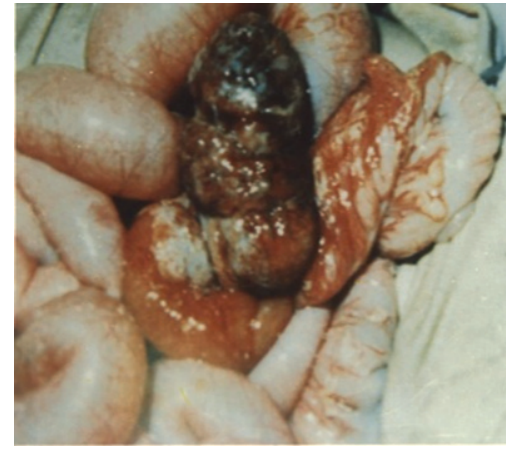

Figure (5e): Intraoperative picture of the same case showing a gangrenous intestine .

air insufflations or hydrostatic enema. This non operative reduction of intussusception has been shown to reduce length of stay, shorten recovery, decrease hospital costs, and decrease the risk of complications related to abdominal surgery. ${ }^{18,19}$ It is known that the earlier the child is managed after the onset of the symptoms, the higher the frequency of successful conservative treatment. The only contraindications to barium enema reduction is peritonitis. ${ }^{16,18}$

At the start of the study, the author did traditional surgical exploration to the first 15 cases, to correlate the clinical, diagnostic investigations and the operative findings. After that, the author started to select the method of management according to the parameters determined from the results of the first 15 cases.

Hydrostatic reduction was attempted in 11 cases all in group 1, with no signs of ischemia, but succeeded in 7 cases only. The other 4 cases were reduced by traditional surgical reduction. The cause of failure of hydrostatic reduction was due to edema of the wall of the intestine. Nonsurgical reduction was not attempted in patients with delayed presentation because of the decreased probability of success and the increased potential for perforation. However, recent data have shown that successful hydrostatic reduction is not influenced by symptom duration. ${ }^{19}$ Many recent studies gave reports above $80 \%$ for non-operative reduction of 
intussusception. ${ }^{20}$

Operative intervention is reserved for cases that were complicated at presentation or failed to respond to pressure treatment.

Laparoscopic reduction was attempted in 7 cases of group 1 with no signs of massive edema or ischemia. Success was achieved in 5 cases only, and failed in 2 cases due to severe resistance with fear of bowel rupture. Abdominal exploration was done and reduction was done manually with some difficulty, with the aid of hot fomentations.

Patients in group 1 were managed by traditional surgical reduction in 22 cases, and surgical resection in 3 cases, while all patients in group 2 were managed by operative reduction in 2 cases and resection in 5 cases. The high incidence of bowel complications in patients with delayed presentation favored routine operative intervention, although, some studies reported successful pressure reduction in some cases presenting after 48 hours, and operative reduction was successful in 2 cases with delayed presentation. ${ }^{21,22}$

At operation, the procedure was chosen according to the findings. In the present study, 5 children who presented after 48 hours of symptoms required bowel resection because of bowel complications. The rate of bowel resection in our cases with delayed presentation was similar to many published reports. ${ }^{23,24}$

The postoperative complications in this study were those for general surgery. The complications were more in patients in group 2 due to the complicated intestine and the bad general condition of the patients. Though the postoperative complication rates differ between the two groups, the absence of significant difference may be due to other factors as the operative technique, tissue handling, antibiotics used and wound management.

Recent reports from developed countries indicate that mortality from childhood Intussusception has steadily declined to under $1 \% .7,25,26$ Most of the reported mortalities in these settings were associated with delayed diagnosis.

In the present report, mortality directly related to intussusception occurred only in one patient in group 2 . This indicates that early diagnosis and good patient care will improve the outcome of Intussusception. Septicemia was the main cause of mortality in our case. So, improving the perioperative care of the patients with aggressive resuscitation, use of more potent antibiotic and postoperative management in an intensive care unit might minimize the mortality. In the long term, improvement in time to diagnosis through maintaining a high index of suspicion, meticulous clinical evaluation, improved access to referral centers, and provision of basic radio diagnostic facilities may help to save these children.

Weihmiller S N et al, 2011, in a study of 38 patients with intussusception, they studied the predictive factors for low risk or high risk patients. Their decision was based on the results of abdominal x-ray (negative or positive), age ( 5 or $>5$ months), diarrhea (present or absent), and bilious vomiting (present or absent). They found a sensitivity of $97 \%$ for these tests. ${ }^{27}$

Kaiser AD et al, 2007, in a study for the current success rate for radiological reduction, requirements for operative intervention, and the effect of delay in presentation on the outcome of intussusception, they reviewed the records for the children treated for intussusception for 15 years. They studied 244 children with intussusception. Their median age was 8.2 months (range, 16 days to 12.7 years). The most common presenting symptoms were emesis $(81 \%)$, hematochezia (61\%), and abdominal pain $(59 \%)$. Contrasted enemas were performed in 190 children, with successful reduction in $46 \%$. Success in reduction was greater if symptom duration was $<24$ hours compared with $>24$ hours ( $59 \%$ vs. $36 \%$ ). One hundred forty children required surgical intervention for Intussusception with 50\% requiring bowel resection. Children with symptom duration $>24$ hours had a greater risk of requiring surgery $(73 \%$ vs. $45 \%)$ and bowel resection $(39 \%$ vs. $17 \%)$ than those with symptoms for $<24$ hours. There were 2 deaths and complications occurring in $19 \%$. Length of 
stay after surgical reduction was 3.9 days and 6.1 days if bowel resection was required. They concluded that delay in presentation decreases success in radiologic reduction and increases risk of operative intervention and bowel resection. ${ }^{7}$

Suzanne Schuh and David E. Wesson, 1987, studied intussusception in children 2 years of age or older. They reviewed the records of 111 children who were treated for intussusception between 1974 and 1984 . They found no complications of attempted barium enema reduction. They reported rate of success for this reduction of $90 \%$ regardless of the duration of illness, even for patients who presented up to 2 weeks after the onset of symptoms. They had 3 deaths related to the original disease and not to intussusception. They concluded that a long history is not in itself a contraindication to hydrostatic reduction, nor is it an automatic indication for laparotomy. ${ }^{28}$

\section{Conclusion:}

1- A considerable proportion of children with intussusception present to the mainstream medical practitioners after 48 hours of symptoms. These patients have a higher incidence of bowel complications requiring resection, and higher mortality rates. Improving the perioperative care of these cases may improve the outcome.

2- Efforts directed at improving time to diagnosis through enhanced referral system and improved diagnostic facilities may optimize the outcome in the long run.

3- Subspecialty and surgeons training for the diagnosis and management of intussusception in children are very important.

4- In intussusceptions, color Doppler allows an interesting evaluation of the degree of bowel ischemia and necrosis and it can change the therapeutic management and appears promising for appreciating the viability of the occluded bowel loops. This recent technique seems to have a place in the strategy of the treatment of such cases.

5- The ability to correlate the diagnostic results and the surgical findings in children with intussusception, has enabled the development of imaging criteria that may indicate which patients may still safely and effectively undergo cautious attempts at barium enema or air reduction. The absence of bowel wall edema, ischemia, bowel obstruction, no peritonitis on ultrasound and Doppler and no free air is seen on radiographic imaging, are all good indications that the bowel can be easily reduced.

\section{References:}

1- Spalding SC, Evans B: Intussusception. Emerg Med 2004; 36 (11) : 12-19.

2- Saxena AK, Seebacher U, Hollwarth ME: Small bowel intussusception: Issues and controversies related to pneumatic reduction and surgical approach. Acta Paediatr 2007; 96: 1651-1654.

3- Huppertz HI, Soriano- Gabaro M, Grimprel E, Franco E, Mezner Z, Desselbeeger U, et al: Intussusception among children in Europe. Paediatr Infect Dis $J$ 2006; 25: 22-29.

4- Khan MJ, Khan K, Kaleem M, Khan M Y, Khan MA, Maroof SA, Khan MT, Uzair M, Ghani M: Retrospective analysis of clinical presentation of children with diagnosed intussusception. JPMI 2007; 21(2): 151-153.

5- Justice FA, Aulist AW, Biness JE: Intussusception: Trends in clinical presentation and management. $J$ Gastroenterol Hepatol 2006; 21: 842-846.

6- Parasher UD, Holman RC, Cummungs KC, Staggs NW, Curns AT, Zimmerman $\mathrm{CM}$, et al: Trends in intussusceptionassociated hospitalizations and deaths among US infants. Pediatrics 2000; 106: 1413-1421.

7- Kaiser AD, Applegate KE, Ladd AP: Current success in the treatment of intussusception in children. Surgery 2007; 142: 469-475.

8- Meier DE, Coin CD, Rescorla FJ, OlaOlorun A, Tarpley JL: Intussusception in children: International perspective. World J Surg 1996; 20: 1035-1039. 
9- Beverly P Wood, John Karani: Imaging in child intussusception. Medscape, 2011.

10-Lim HK,Bae SH, Lee KH, Yoon GS: Assessment of reducibility of ileocolic intussusception in children: Usefulness of color Doppler sonography. Radiology 1994; 191: 781.

11-Pracros JP, Tran-Minh VA, Morin De Finfe $\mathrm{CH}$ : Acute intestinal intussusception in children, contribution of ultrasonography. Ann Radiology 1987; 30 : 525.

12-Ugwu BT, Legbo JN, Dakum NK, Yiltok SJ, Mbah N, Uba FA. Childhood intussusception: A 9-year review. Ann Trop Paediatr 2000; 20: 131-135.

13-Saxena AK, Hollwarth ME: Factors influencing management and comparison of outcomes in pediatric intussusceptions. Acta Paediatr 2007; 96: 1199-1202.

14-Latipov R, Khudoyorov R, Flem E: Childhood intussusception in Uzbekistan: Analysis of retrospective surveillance data. BMC Pediatrics 2011; 11: 22.

15-Ameh EA: The morbidity and mortality of laparotomy for uncomplicated intussusception in children. West Afr $J$ Med 2002; 21: 115-116.

16-Van Heek NT, Aronson DC, Halimum EM, Soewarno R, Molenaar JC, Vos A: Intussusception in a tropical country: Comparison among patient population in Jakarta, Jogyakarta, and Amsterdam. J Pediatr Gastroenterol Nutr 1999; 29: 402-405.

17-Caneiro PM, Kisusi DM: Intussusception in children seen at Muhimbili National Hospital, Dar es Salaam. East Afr Med J 2004; 81: 439-442.

18-Bai YZ, Ou RB, Wang GD, Zhang KR, Li Y,Huang Y et al: Ultrasound- guided hydrostatic reduction of intussusception by saline enema: A review of 5218 cases in 17 years. Am J Surg 2006; 192: 273-275.

19-Van den Ende ED, Allema JH, Hazebroek FW, Breslau PJ: Success with hydrostatic reduction of intussusception in relation to duration of symptoms. Arch Dis Chil 2005; 90 (10): 1071-1072.

20-Somme S, To T, Langer JC: Factors determining the need for operative reduction in children with intussusception: A population- based study. Journal of Pediatric Surgery 2006; 41: 1014-1019.

21-Fragoso AC, Campos M, Tavares C, CostaPereira A, Estevao- Costa J: Pneumatic reduction of childhood intussusception: Is prediction of failure important? J Pediatr Surg 2007; 42: 1504-1508.

22-Okuyama H, Nakai H, Okada A: Is barium enema reduction safe and effective in patients with a long duration of intussusception? Pediatr Surg Int 1999; 15: 105-107.

23-Ko SF, Lee TY, Ng SH, Wan YL, Chen MC, Tiao MM, et al: Small bowel intussusception in symptomatic pediatric patients: Experience with 19 surgically proven cases. World J Surg 2002; 26: 438-443.

24- Ein SH, Alton D, Palder SB, Shandling B, Stinger D: Intussusception in the 1990s: Has 25 years made a difference? Pediatr Surg Int 1997; 12: 374-376.

25-Buettcher M, Baer G, Bonhoeffer J, Schaad UB, Heininger U. Three - year surveillance of intussusception in children in Switzerland. Pediatrics 2007; 120: 473-480.

26-Blanch AJ, Perel SB, Acworth JP. Paediatric intussusception: Epidemiology and outcome. Emerg Med Australs 2007; 19: 45-50.

27-Weihmiller SN, Buonomo C, Bachur $\mathrm{R}$ : Risk stratification of children being evaluated for intussusception. $J$ Watch Emergency Med 2011; 25: 4-41.

28-Schuh S, Wesson DE: Intussusception in children 2 years of age or older. CMAJ 1987; 138 (3): 269-272. 